\title{
Low-Fouling Electrosprayed Hemoglobin Nanoparticles with Antioxidant Protection as Promising Oxygen Carriers
}

Liu, Xiaoli; Jansman, Michelle M.T.; Thulstrup, Peter W.; Mendes, Ana C.; Chronakis, Ioannis S.; HostaRigau, Leticia

Published in:

Macromolecular Bioscience

Link to article, DOI:

10.1002/mabi.201900293

Publication date:

2020

Document Version

Peer reviewed version

Link back to DTU Orbit

Citation $(A P A)$ :

Liu, X., Jansman, M. M. T., Thulstrup, P. W., Mendes, A. C., Chronakis, I. S., \& Hosta-Rigau, L. (2020). LowFouling Electrosprayed Hemoglobin Nanoparticles with Antioxidant Protection as Promising Oxygen Carriers. Macromolecular Bioscience, 20(2), [1900293]. https://doi.org/10.1002/mabi.201900293

\section{General rights}

Copyright and moral rights for the publications made accessible in the public portal are retained by the authors and/or other copyright owners and it is a condition of accessing publications that users recognise and abide by the legal requirements associated with these rights.

- Users may download and print one copy of any publication from the public portal for the purpose of private study or research.

- You may not further distribute the material or use it for any profit-making activity or commercial gain

- You may freely distribute the URL identifying the publication in the public portal 


\title{
Low-fouling Electrosprayed Hemoglobin Nanoparticles with Antioxidant Protection as Promising Oxygen Carriers
}

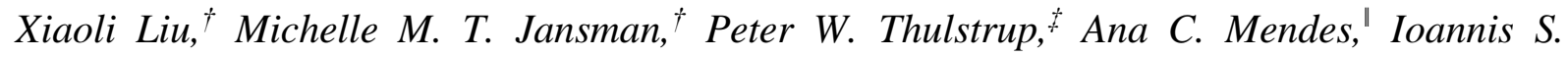
Chronakis," and Leticia Hosta-Rigau, ${ }^{\dagger, *}$

Dr X. Liu, M. M. T. Jansman, Prof. L. Hosta-Rigau

DTU Health Tech, Center for Nanomedicine and Theranostics, Technical University of Denmark, Nils Koppels Allé, B423, 2800 Kgs. Lyngby, Denmark

E-mail: leri@dtu.dk

Prof P. W. Thulstrup

Department of Chemistry, University of Copenhagen, Universitetsparken 5, 2100

Copenhagen, Denmark

Prof A. C. Mendes, Prof I. S. Chronakis

DTU Food, Technical University of Denmark, Kemitorvet, B202, 2800 Kgs. Lyngby, Denmark

Keywords: electrospray, hemoglobin-based oxygen carriers, hemoglobin nanoparticles, poly(dopamine), oxidative toxicity

\begin{abstract}
Despite all the attempts to create advanced hemoglobin (Hb)-based oxygen carriers (HBOCs) employing an encapsulation platform, major challenges including attaining a high $\mathrm{Hb}$ loading and long circulation times, still need to be overcome. Herein the fabrication, for the first time, of nanoparticles fully made of $\mathrm{Hb}(\mathrm{Hb}-\mathrm{NPs})$ employing the electrospray technique is reported. The Hb-NPs are then coated by antioxidant and self-polymerized poly(dopamine) (PDA) to minimize the conversion of $\mathrm{Hb}$ into non-functional methemoglobin (metHb). The PDA shell is further functionalized with poly(ethylene glycol) (PEG) to achieve stealth properties. Our results demonstrate that the as-prepared $\mathrm{Hb}-\mathrm{NPs}$ are hemo- and biocompatible while offering antioxidant protection and decreasing the formation of metHb. Additionally, decoration with PEG results in decreased protein adsorption onto the Hb-NPs surface, suggesting a prolonged retention time within the body. Finally, the Hb-NPs also preserve the reversible oxygen-binding
\end{abstract}


and releasing properties of $\mathrm{Hb}$. All in all, within this study, a novel HBOCs with high $\mathrm{Hb}$ content is fabricated and its potential as an artificial blood substitute is evaluated.

\section{Introduction}

Blood transfusions, which typically consist on the administration of packed red blood cells (RBCs), are currently an essential and life-saving clinical procedure. They are widely employed, among others, in the treatment of anemia, various blood disorders such as hemophilia, and side-effects of tumor chemotherapy. ${ }^{[1,2]}$

However, RBCs transfusions have several limitations including the potential mismatch during transfusion due to the RBCs membrane antigens, the risks of viral or bacterial transmission (e.g., HIV, malaria or hepatitis) and the limited availability due to their short shelf-life (1 day at room temperature and 42 days in refrigerated conditions). ${ }^{[3,4]}$ Such limitations and risks are even more severe in some developing countries; where blood donations are either still not routinely tested for transmissible pathogens or, when tested, the laboratories lack sufficient quality assurance. $^{[5]}$

As such, great efforts have been devoted to the development of artificial blood substitutes that are free from the above-mentioned risks. In particular, the development of hemoglobin-based oxygen carriers (HBOCs), which make use of the main functional component of biological RBCs, have received a great deal of attention. ${ }^{[6]}$ Hemoglobin $(\mathrm{Hb})$ is a tetrameric protein containing two $\alpha$ and two $\beta$ subunits, each of which incorporates an iron-containing heme group that is able to bind oxygen with high affinity. ${ }^{[7]}$ However, unmodified stroma-free $\mathrm{Hb}$ dissociates into the two subunits ( $\alpha$ and $\beta$ ), causing severe adverse effects such as renal toxicity and vasoconstriction. ${ }^{[8]}$ Approaches to overcome $\mathrm{Hb}$ 's breakdown include the modification of $\mathrm{Hb}$ by intra- and intermolecular cross-linking,,$^{[9,10]}$ polymerization, ${ }^{[11]}$ or polymer conjugation. ${ }^{[12]}$ However, so far, persistent safety concerns regarding the toxicity of HBOCs in phase II and phase III clinical trials have hampered the development of a clinically viable 
oxygen carrier. ${ }^{[13,14]}$ Mechanisms contributing to the toxicity of these HBOCs are mainly attributed to their permeation in between the endothelial cells lining the capillary walls and into the smooth muscle tissue. There they act as a nitric oxide (NO) scavenger. ${ }^{[15]}$ Since NO is an important vasodilator, ${ }^{[16]}$ NO scavenging results in vasoconstriction, the subsequent cardiovascular problems and increased mortality rates. ${ }^{[17]}$

Thus, an approach to solve current limitations of HBOCs would be the use of an encapsulation platform. Hb's encapsulation within a carrier shell can provide a suitable environment to maintain its functionality while, at the same time, preventing tetramer dissociation as well as Hb's extravasation into the smooth muscle tissue. As such, recent research on HBOCs has mainly focused on Hb's encapsulation within several carriers including liposomes, ${ }^{[18]}$ polymersomes ${ }^{[19]}$ or polymer capsules. ${ }^{[20]}$ Despite the progress, achieving a high $\mathrm{Hb}$ loading within a well-defined biocompatible and biodegradable structure still remains as a significant challenge. To achieve an oxygen carrying capacity similar to that of blood, a high loading of $\mathrm{Hb}$ within the carriers is crucial since, within biological RBCs, Hb comprises about $96 \%$ of the dry weight.

Herein, we employed a facile and controllable way to fabricate particles fully made of $\mathrm{Hb}$ and explored their potential as oxygen carriers. We prepared $\mathrm{Hb}$ nanoparticles (Hb-NPs) by the electrospray technique (Scheme 1). This technique makes use of an electric field to disperse or break up a liquid to generate monodisperse droplets in a fast and one-step procedure. The size of the resulting particles can be efficiently tailored from tens of nanometers to hundreds of micrometers by the right choice of the operation parameters. ${ }^{[21]}$ Furthermore, the electrospray technique allows for the fabrication of biomacromolecule-based particles (such as proteins, DNA or carbohydrates) without loss of their bioactivity. ${ }^{[21-25]}$

Another remaining concern when developing oxygen-carriers is their insufficient circulation times, which are much shorter than those of biological RBCs. Macrophages in the mononuclear 
phagocyte system (MPS) can efficiently engulf foreign particles, a process that mainly depends on the size and surface characteristics of the particles. ${ }^{[26]}$ Thus, while Hb carriers in the size range of $1-3 \mu \mathrm{m}$ are expected to be strongly phagocytosed, ${ }^{[27]}$ carriers should be larger than 100 $\mathrm{nm}$ to avoid the particles extravasation in between the endothelial gaps. Regarding surface coatings, the golden standard strategy to evade the MPS consists of surface PEGylation. ${ }^{[28]}$ As such, the size of the electrosprayed $\mathrm{Hb}-\mathrm{NPs}$ was fine-tuned to $400 \mathrm{~nm}$ and their surface decorated by biocompatible PEG.

Prior to PEGylation, the Hb-NPs are coated with polydopamine (PDA), which is an effective adhesive material that can spontaneously form a continuous coating layer on a wide variety of surfaces. ${ }^{[2]}$ Furthermore, PDA has antioxidant properties which are crucial to minimize the conversion of $\mathrm{Hb}$ into non-functional methemoglobin (metHb). ${ }^{[30]}$ Additionally, PEGylation of the Hb-NPs (Hb/PEG-NPs) is easily achievable by reaction of the PDA coating with the amino groups of the poly-L-lysine-graft-PEG (PLL-g-PEG) copolymer. Finally, we assess the potential of the Hb/PEG-NPs as oxygen-carriers in terms of structural integrity, oxygen-binding and releasing properties together with hemo- and biocompatibility.

This report on the first electrosprayed NPs fully made of $\mathrm{Hb}$ opens the door for the development of HBOCs able to fulfil the high oxygen demands similar to native RBCs.

\section{Results and Discussion}

\subsection{Fabrication of $\mathrm{Hb}-\mathrm{NPs}$}

Benefits of HBOCs over donor blood include universal compatibility, availability, long-term storage and low risk of infection. To achieve high oxygen demands similar to biological RBCs, we prepared nanoparticles fully made of hemoglobin (Hb-NPs) employing the electrospray technique. Electrospray becomes an ideal technique for the fabrication highly ordered nanostructures with preserved bio-functionality since, ${ }^{[25,31]}$ as a result of the electric forces, it

facilitates the re-arrangement of (bio)macromolecules. ${ }^{[32]}$ Bovine $\mathrm{Hb}$ was the material of choice 
to avoid the need for the 2,3-diphosphoglycerate allosteric effector, which is required for the native activity of human $\mathrm{Hb}^{[33]}$ In contrast, bovine $\mathrm{Hb}$ only requires chloride ion for biological operation, ${ }^{[34]}$ which is already present in blood and can be further provided by buffers like PBS or Ringer's lactate solution. As such, a solution of bovine Hb in Milli-Q (MQ) water was electrosprayed and the resulting Hb-NPs were collected either with a stationary plate (Hb-NP1) or an ethanol (EtOH) bath (Hb-NP2) at room temperature and ambient humidity. Scanning electron microscopy (SEM) images revealed that the obtained particles (both $\mathrm{Hb}-\mathrm{NP} 1$ and $\mathrm{Hb}$ NP2) are mostly monodispersed particles and free from large aggregates (Figure 1a), while Hb-NP2 is more even compared Hb-NP1. However, Hb-NP1 emerges irregular but partly spherical shaped, while Hb-NP2 displays a spherical morphology. Measured by nanoparticle tracking analysis (NTA), the average size is $374 \pm 188 \mathrm{~nm}$ and $482 \pm 215 \mathrm{~nm}$ for $\mathrm{Hb}-\mathrm{NP} 1$ and $\mathrm{Hb}-\mathrm{NP} 2$, respectively. This submicron size for both $\mathrm{Hb}-\mathrm{NPs}$ is an important feature since the therapeutic window for HBOCs lays between $100 \mathrm{~nm}$ and $1 \mu \mathrm{m}$. While particles larger than 5 $\mu \mathrm{m}$ can obstruct the microcirculation, particles in the size range of $1-3 \mu \mathrm{m}$ are expected to be strongly depleted by the MPS. In addition, particles smaller than $100 \mathrm{~nm}$ can extravasate through the endothelial cells lining the blood vessels, HBOCs. ${ }^{[35]}$

Interestingly, both types of Hb-NPs became insoluble on water and, thus, could be dispersed in MQ. This result is not surprising since, the charge accumulated onto the surface of the Hb's droplets during the electrospray process, could alter the conformation of the proteins structure. ${ }^{[36]}$ This effect particularly affects the biomolecules located on the NP's surface. We hypothesize that this change in solubility could be a result of the exposure of the hydrophobic binding pockets for the $\mathrm{Hb}$ molecules located onto the NP's surface. The effect of this hypothetical conformation change will be evaluated in the following sections. 
The stability of the NPs following freeze-drying and storage as a dried powder for several weeks was also evaluated. Figure S1, Supporting Information, shows how both types of NPs emerge mainly monodispersed and free from large aggregates following re-suspension in MQ.

\subsection{Hb-NPs Surface Functionalization}

To avoid Hb's leakage from the particles, we employed a PDA coating by the selfpolymerization of dopamine (DA) in basic conditions. ${ }^{[29]}$ PDA has scarcely any chemical reaction with Hb's functional groups while also allowing for post-functionalization (e.g., PEGylation) by its ability to react via Schiff base and/or Michael addition with thiols and amines. ${ }^{[37]}$ Furthermore, PDA can also offer antioxidant protection thus minimizing the conversion of $\mathrm{Hb}$ into non-functional metHb. ${ }^{[30]}$

The PDA coating was fabricated by the self-polymerization of DA in $10 \mathrm{mM}$ TRIS at $\mathrm{pH} 8.5$ (TRIS buffer) for $0.5 \mathrm{~h}$. The successful DA polymerization was corroborated by the color change of the suspension into the characteristic black appearance (Figure 1b, inset). Following several washes to remove the unreacted DA and resuspension in MQ water ( $\mathrm{pH}$ 6.8) to stop the reaction, the PDA-coated particles (Hb/PDA-NP1 and Hb/PDA-NP2) were imaged by SEM. Figure 1b shows no apparent change in terms of morphology or size as compared to the uncoated counterparts. To render the Hb-NPs with low-fouling properties, we further functionalized the carriers' surface with amine-containing PEG chains. Biocompatible PLL-gPEG was bound to the Hb/PDA-NPs surface with the aim to substantially increase their circulation time in vivo. The SEM images (Figure 1c) show no noticeable changes as compared to the bare or PDA-coated counterparts (Figure 1a, and 1b, respectively). Additionally, no differences could be observed depending on the collection method. The functionalization of the $\mathrm{Hb}-\mathrm{NPs}$ surface was also assessed by zeta $(\zeta)$-potential measurements (Figure $1 \mathrm{~d})$. Although free $\mathrm{Hb}$ has a negative $\zeta$-potential of $-29.6 \mathrm{mV}$, both electrosprayed $\mathrm{Hb}$-NPs display a positive $\zeta$-potential of 27.5 and $26.9 \mathrm{mV}$ for $\mathrm{Hb}-\mathrm{NP} 1$ and $\mathrm{Hb}-\mathrm{NP} 2$, respectively. This is not surprising 
since the electrospray process influences the ionisable groups which could, in turn, affect the overall surface charge of the generated particles. ${ }^{[38]}$ For the as-prepared Hb-NPs, we speculate that the positive charge mode of the particles is due to the protonation of the basic amino acid side chains of $\mathrm{Hb}$. Upon DA polymerization into PDA, a decrease in $\zeta$-potential of 6.5 and 3.2 $\mathrm{mV}$ for $\mathrm{Hb} / \mathrm{PDA}-\mathrm{NP} 1$ and $\mathrm{Hb} / \mathrm{PDA}-\mathrm{NP} 2$, respectively, was observed. The successful coupling of the PEG layer onto the NPs surface was then confirmed by an increase in $\zeta$-potential of $\sim 12.7$ and $\sim 15.5 \mathrm{mV}$ for $\mathrm{Hb} / \mathrm{PEG}-\mathrm{NP} 1$ and $\mathrm{Hb} / \mathrm{PEG}-\mathrm{NP} 2$, respectively.

Next, we assessed the PEGylation effect of the coated NPs (Hb/PEG-NP1 and Hb/PEG-NP2) in terms of protein adsorption. Functionalization of the carriers surface with hydrophilic polymers such as PEG has been widely used in the drug delivery field to shield the surface from opsonization. ${ }^{[39]}$ Upon intravenous administration, the nanocarriers will be recognized and cleared by the MPS due to opsonin accumulation on their surface. ${ }^{[40]}$ Since serum albumin and immunoglobulin $\mathrm{G}(\mathrm{IgG})$ are the two most abundant proteins in blood, the evaluation of the PEGylation effect was assessed in terms of inhibition of absorption of fluorescein isothiocianate (FITC)-labelled bovine serum albumin (BSA-FITC) and IgG (IgG-FITC). While IgG is known to be a potent opsonin promoting recognition and uptake by the MPS, albumin has been suggested to promote a dypsonic effect. ${ }^{[41]}$ However, albumin can be easily replaced by other proteins exerting an opsonic effect. ${ }^{[42]}$ Thus, bare Hb-NPs and PEGylated Hb/PEG-NPs were incubated with either BSA-FITC or IgG-FITC at $37{ }^{\circ} \mathrm{C}$ for $4 \mathrm{~h}$. The binding of the fluorescent proteins onto the NPs was evaluated by flow cytometry. The results were normalized to the mean fluorescence intensity (nMFI) of bare NPs (Hb-NP1 and Hb-NP2) incubated with BSAFITC and IgG-FITC (Figure 2). The results show a different PEGylation effect depending on the collection method employed to fabricate the Hb-NPs (i.e., stationary plate and EtOH bath for $\mathrm{Hb}-\mathrm{NP} 1$ and $\mathrm{Hb}-\mathrm{NP} 2$, respectively). While for Hb-NP1 a significant decrease in nMFI $(\sim 24 \%)$ is only observed for PEGylated particles incubated with BSA-FITC; for Hb-NP2 the 
outcome is different. PEG functionalization results in a decrease in nMFI of $\sim 56 \%$ and $\sim 69 \%$ upon incubation with BSA-FITC and IgG-FITC, respectively.

Although, at this point of time, it is not known what causes Hb/PEG-NP2 to display enhanced low-fouling properties as compared to Hb/PEG-NP1, it is noted that the two collection methods rendered particles of slightly different morphologies (i.e., irregular-shaped for Hb-NP1 and smooth spherical for Hb-NP2). We speculate that the difference in surface roughness could lead to an enhanced absorption of the fluorescently labelled proteins for Hb-NP1.

\subsection{Preservation of Hb's Functionality}

\subsubsection{Fourier-Transform Infrared (FTIR) Spectroscopy}

The oxygen-carrying capacity is the most important aspect when developing HBOCs. Thus, preservation of the Hb's structure within the different carriers is crucial to secure oxygen transport. The effect of the electrospray process on Hb's chemical structure was analyzed by FTIR spectroscopy. Figure 3 shows FTIR spectra of native $\mathrm{Hb}$ and the as-prepared NPs with the different coatings (Hb-NPs, Hb/PDA-NPs and Hb/PEG-NPs) collected in a stationary plate (NP1, Figure 3a) or in an EtOH bath (NP2, Figure 3b). The results show the characteristic bands at $1650 \mathrm{~cm}^{-1}$ and $1534 \mathrm{~cm}^{-1}$ for all the samples. For proteins, the band between $1600-1700$ $\mathrm{cm}^{-1}$ originates from the stretching vibrations of the $\mathrm{C}=\mathrm{O}$ (Amide $\mathrm{I}$ ) while the band between $1510-1580 \mathrm{~cm}^{-1}$ derives from in-plane $\mathrm{N}-\mathrm{H}$ bending and $\mathrm{C}-\mathrm{N}$ stretching vibrations (Amide II). The comparison of the FTIR spectra of native $\mathrm{Hb}$ and the Hb-NPs, which shows almost identical absorption bands for Amide I and Amide II, suggests preservation of the $\mathrm{Hb}$ 's chemical structure after the electrospray process. Additionally, after surface modification with PLL-gPEG, the Hb/PEG-NPs show the characteristic absorption peak of the C-O-C stretching vibration at $1100 \mathrm{~cm}^{-1}$. Thus, in agreement with the $\zeta$-potential measurements, FTIR spectroscopy indicates successful PEGylation of the NPs (Figure 3c).

\subsubsection{Circular Dichroism (CD) Studies}


Since the secondary structure of proteins is crucial to their function, preservation of this structure can be used to presume protein activity. ${ }^{[43]}$ The retention of Hb's structure after the electrospray process was assessed by far UV-CD spectroscopy, a technique that has been widely employed to monitor protein secondary structure. ${ }^{[44]}$ The UV-CD spectra were measured in the range from 190 to $260 \mathrm{~nm}$ for native $\mathrm{Hb}$ and for $\mathrm{Hb}-\mathrm{NPs}$ depending on the collection method (NP1: stationary plate or NP2: EtOH bath). The samples were monitored in identical buffer conditions to evaluate potential structural changes in $\mathrm{Hb}$. Figure 4 shows the typical absorption peaks of the $\alpha$-helix structure for free $\mathrm{Hb}$ and both types of $\mathrm{Hb}-\mathrm{NPs}$ (maximum at $194 \mathrm{~nm}$ and double minima at 208 and $222 \mathrm{~nm}$ ). However, a decrease in the intensity of the peak was observed for the Hb-NPs as compared to the free $\mathrm{Hb}$. This decrease in intensity might be attributed to the absorbance of the NPs in this wavelength range or to a partial loss of $\alpha$-helix structure. Such decrease in intensity was more pronounced for the NPs collected with the EtOH bath (Hb-NP2, pink line) as compared to the NPs collected with a stationary plate (Hb-NP1, green line). We hypothesized that the enhanced loss of $\alpha$-helix structure for Hb-NP2 might be induced by the EtOH bath. As reported elsewhere, EtOH could cause a complete denaturation of proteins when reaching a concentration $50 \%(\mathrm{v} / \mathrm{v}) .{ }^{[45]}$

Upon normalizing the CD spectra to the $222 \mathrm{~nm}$ peak, the CD spectra of both Hb-NPs were very similar to the spectrum of free $\mathrm{Hb}$ (Figure 4, right side). This result indicates that most of all the detectable $\mathrm{Hb}$ within the particles maintained its secondary structure. The minor differences seen in the normalized CD spectra could indicate that a fraction of the $\mathrm{Hb}$ in both NPs has a perturbed folding, but might also reflect effects due to environmentally-induced wavelength shifts, light scattering, and absorption flattening in the particles. ${ }^{[46]}$

As discussed in section 2.3.1, Hb molecules in the NPs surface might re-arrange forming a supramolecular layer; losing their secondary structure while making the NPs insoluble in water. 
This supramolecular layer could, in turn, protect the inner $\mathrm{Hb}$ molecules from misfolding and denaturation.

\subsubsection{Oxygen Binding and Releasing Properties}

We next investigated whether the Hb entrapped within the NPs maintained its native bioactivity. To do so, the ability of the Hb-NPs in binding and releasing oxygen was evaluated by spectral analysis. Since $\mathrm{Hb}$ can be easily oxidized into metHb, which cannot bind to molecular oxygen $\left(\mathrm{O}_{2}\right)$, we first incubated the $\mathrm{Hb}-\mathrm{NPs}$ in a sodium dithionite solution to reduce metHb back into functional oxygenated-Hb (oxy-Hb). Next, nitrogen $\left(\mathrm{N}_{2}\right)$ and $\mathrm{O}_{2}$ gas were successively flowed over the NPs collected by a stationary plate (Hb-NP1 and Hb/PEG-NP1) or an EtOH bath (HbNP2 and Hb/PEG-NP2). The obtained deoxygenated-Hb (deoxy-Hb) and oxy-Hb-based NPs were evaluated by UV-vis spectroscopy. Figure 5 shows the initial characteristic peaks of oxy$\mathrm{Hb}$ at $\sim 408 \mathrm{~nm}$ (Soret peak), $\sim 536$ and $\sim 577 \mathrm{~nm}$ (dark red lines) for bare Hb-NPs and PEGylated Hb/PEG-NPs independent of the collection method (NP1: stationary plate method (Figure 5a) or NP2: EtOH bath method (Figure 5b)). Next, $\mathrm{N}_{2}$ gas was flowed over the oxy-Hb-based NPs (Hb-NPs and Hb/PEG-NPs) which resulted on a shift of the Soret peak to $428 \mathrm{~nm}$ and the disappearance of the peaks at $\sim 536$ and $\sim 577 \mathrm{~nm}$ (dark green lines). The appearance of the characteristic deoxy- $\mathrm{Hb}$ peak demonstrates the ability of the entrapped $\mathrm{Hb}$ to release oxygen. Next, $\mathrm{O}_{2}$ gas was fed for $10 \mathrm{~min}$ and the characteristic deoxy-Hb Soret peak shifted from 428 $\mathrm{nm}$ back to a value closer to $408 \mathrm{~nm}$ (red lines). Additionally, the peaks at 536 and $577 \mathrm{~nm}$ could be observed again for some of the samples. This indicates the conversion of the entrapped $\mathrm{Hb}$ from the deoxy-Hb to the oxy-Hb state. The movement of characteristic peaks showed in. Figure S2 (Supporting Information). It is important to note that the decrease in the intensity of the absorbance peak is due to loss of sample in the process of gas flow, which enhances the solvent evaporation and thus speeding up the drying out of the Hb-NPs and attaching on the top of the container. A second $\mathrm{N}_{2}$ and $\mathrm{O}_{2}$ gas cycles were successfully conducted demonstrating that both bare (Hb-NPs) and PEGylated (Hb/PEG-NPs) NPs could reversibly bind and release 
oxygen. Although there is a change on the wavelength of absorbance peak for both Hb-NPs and $\mathrm{Hb} / \mathrm{PEG}-\mathrm{NPs}$ following flowing with $\mathrm{O}_{2}$ and $\mathrm{N}_{2}$, this change is more pronounced for $\mathrm{Hb}$ - and $\mathrm{Hb} / \mathrm{PEG}-\mathrm{NP} 1$, thus suggesting better oxygen binding and releasing properties for NPs employing a stationary plate as collecting method. This is in agreement with the CD spectra results which showed enhanced preservation of $\alpha$-helix structure for Hb-NP1.

Nonetheless, these results demonstrate that even after the electrospray process, surface modification with PDA and subsequent functionalization with PEG, both types of NPs still show oxygen-carrying ability, which is the most important feature of HBOCs.

\subsubsection{Antioxidative Protection of $\mathrm{Hb}$ by the PDA Coating}

The oxidative protection of $\mathrm{Hb}$ against hydrogen peroxide $\left(\mathrm{H}_{2} \mathrm{O}_{2}\right)$ by the PDA coating was assessed by UV-vis. In particular, we monitored the characteristic absorption Soret peak of oxy$\mathrm{Hb}$ upon incubation with $\mathrm{H}_{2} \mathrm{O}_{2}$ at different time points (Figure S3, Supporting Information). The results, which have been normalized to the height of the Soret peak before the addition of $\mathrm{H}_{2} \mathrm{O}_{2}$, are illustrated in Figure 6. As expected, the highest decrease in Soret peak height is observed for uncoated Hb-NPs independent of the collection method (NP1: stationary plate method (Figure 6a) or NP2: EtOH bath method (Figure 6b)). While, for NP2, the additional surface modification with PEG does not have an influence on metHb content (i.e., both Hb/PDA-NP2 and Hb/PEG-NP2 cause a reduction of Soret peak of only 20\% after 15 min), the results are different for NP1. While Hb/PEG-NP1 only causes a $20 \%$ decrease in Soret peak height after $15 \mathrm{~min}$, the decrease in Soret peak height by Hb/PDA-NP1 is much more pronounced ( 40\% after $15 \mathrm{~min})$.

We speculate that the differences in the antioxidant properties of the PDA coating depending on the collection method could be due to the different shapes of the NPs. The slightly peanutshaped Hb/PDA-NP1 display a higher surface area as compared to the more spherical Hb/PDANP2, which will result in thinner and thus less protective PDA layer. Nonetheless, is worth 
noticing that the PDA coating minimized the oxidation of $\mathrm{Hb}$ into metHb for both studied conditions.

\subsection{Interaction with Cells}

The next step was to evaluate the biocompatibility of the as-prepared HBOCs in terms of hemocompatibility and cell viability as a first step towards the development of safe and effective blood substitutes.

\subsubsection{Hemolysis Rate}

Hemocompatibility is crucial for any intravenously administered carrier. According to ISO/TR 7406, if the hemolysis rate (extent of hemolysis via the quantification of free $\mathrm{Hb}$ in the mixed solution) is less than 5\%, the biomaterials are considered non-hemolytic. ${ }^{[4]}$ As shown in Figure 7ai, the Hb-NPs, independent of the surface coating (Hb-NP, Hb/PDA-NP, Hb/PEG-NP) or the collection method (NP1: stationary plate or NP2: EtOH bath) showed a hemolysis rate well within $1 \%$, thus implying good hemocompatibility. Figure $7 \mathrm{~b}$ shows photographic images of the positive control ( $100 \%$ lysis), the negative control ( $0 \%$ lysis), and the different NPs depending on the surface coating and the collection method. Only for the positive control red free $\mathrm{Hb}$ can be observed in the suspensions. Importantly, the blood cells retained their shape after incubation with the different NPs (Figure S4, Supporting Information). As expected, due to lysis no blood cells could be observed in the positive control.

\subsubsection{Cell Viability}

The biocompatibility of the NPs was also evaluated in terms of in vitro cell viability on human umbilical vein endothelial cells (HUVEC) and mice macrophages RAW 264.7 cells. While endothelial cells are the cells lining our blood vessels, circulating macrophages are the first line of defense of the human body against intruding pathogens. Figures $7 \mathrm{~b}$ and $\mathrm{c}$ demonstrate that the different NPs did not produce significant decrease in cell viability on either HUVEC (Figure 7bi) or RAW 264.7 (Figure 7ci) cells for 4 h. However, upon incubation for $24 \mathrm{~h}$, the results were different for both cell lines (Figure 7bii and 7cii). For RAW 264.7 cells, incubation with 
the NP1s (Hb-NP1, Hb/PDA-NP1 and Hb/PEG-NP1) resulted in an increase in cell viability (up to $20 \%$ ) (Figure 7cii). Interestingly, incubation of the different NPs with HUVEC cells resulted in an increase in cell viability for almost all the NPs independent of surface coating and preparation method (NP1: stationary collector and NP2: EtOH bath) (Figure 7bii). This is not surprising since $\mathrm{Hb}$-based particles acting as oxygen releasing microcarriers have prior history showing increasing cells viability, ${ }^{[48]} \mathrm{a}$ fact of utmost importance for in vitro tissue engineering applications where oxygen supply becomes crucial during the fabrication of large tissue analogues due to the "size-barrier effect". In addition, previous investigations have proven that a PDA outer layer can also promote cell proliferation. ${ }^{[49],[50]}$ Importantly, those results suggest that the as-prepared $\mathrm{Hb} / \mathrm{PEG}-\mathrm{NPs}$ could be a potential oxygen carrier because of their excellent biocompatibility.

\section{Conclusion}

We have presented a new method to prepare NPs fully made of $\mathrm{Hb}$. By making use of the electrospray technique and two different collecting methods (i.e., a stationary plate or an EtOH bath), Hb-based NPs of slightly different morphology have been fabricated.

Both types of Hb-NPs have a size $\sim 400 \mathrm{~nm}$ and, as such, they are expected to be able to avoid extravasation through the blood vessel wall and, thus, circumvent vasoconstriction.

Coating with PDA results on a decrease of Hb's oxidation to non-functional metHb. Biocompatible PEG is used to further modify the Hb-NPs and results in less protein deposition onto the surface of the particles. This suggests protection of the Hb-NPs against immune response following in vivo administration. Both types of Hb-NPs are able to bind and release oxygen, which is the most important feature for HBOCs. However, Hb-NPs collected with the stationary plate display enhanced oxygen-binding and releasing properties as compared to $\mathrm{Hb}$ NPs collected with an EtOH bath. This fact is attributed to the denaturing effects of EtOH. Nonetheless the as-prepared $\mathrm{Hb}-\mathrm{NPs}$ may have potential to be useful artificial oxygen carriers in the future. 


\section{Experimental Section}

Materials: Fresh bovine blood (Product No. 77667) was obtained from SSI Diagnostica A/S (Hillerød, Denmark). Ethanol (EtOH), dopamine (DA), sodium chloride ( $\underline{\mathrm{NaCl}})$, toluene, tris(hydroxymehtyl)aminomethane (TRIS), poly-L-lysine (PLL) (Mw 15 - $30 \mathrm{kDa}$ ), sodium dithionite (SDT), 4-(2-hydroxyethyl) piperazine-1-ethane-sulfonic acid (HEPES), fluorescein isothiocyanate (FITC), FITC-labelled immunoglobulin G (IgG-FITC), bovine serum albumin (BSA), phosphate buffered saline (PBS), Dulbecco's Modified Eagle's Medium-high glucose (DMEM D5796), penicillin/streptomycin, fetal bovine serum (FBS), hydrogen peroxide $\left(\mathrm{H}_{2} \mathrm{O}_{2}\right)$, trypsin and the human umbilical vein endothelial cell line (HUVEC) were purchased from Sigma-Aldrich (Saint Louis, MO, USA). $\alpha$-Methoxy- $\omega$-carboxylic acid succinimidyl ester poly(ethylene glycol) (Me-PEG-NHS, MwPEG = $2000 \mathrm{Da})$ was purchased from Iris Biotech GmbH (Marktredwitz, Germany). Endothelial Cell Medium (ECM) Kit supplemented with 5\% FBS and 1\% endothelial cell growth supplements was purchased from Innoprot (Derio-Biskaia, Spain). PrestoBlue Cell Viability Reagent, and Pierce Bicinchoninic Acid (BCA) Protein Assay Kit were obtained from Thermo Fisher Scientific (Waltham, MA). The macrophage cell line RAW 264.7 was obtained from European Collection of Authenticated Culture Collections (ECACC, Wiltshire, UK).

TRIS buffer is composed of $10 \mathrm{mM}$ TRIS (pH 8.5); HEPES buffer is composed of $10 \mathrm{mM}$ HEPES and $150 \mathrm{mM} \mathrm{NaCl}(\mathrm{pH}$ 7.4). All buffers were prepared with ultrapure water (Milli-Q (MQ), gradient A 10 system, TOC < 4 ppb, resistance 18 MV cm, EMD Millipore, USA).

BSA was fluorescently labelled with FITC (BSA-FITC) as previously reported. ${ }^{[51]}$ Briefly, a solution containing $3.7 \mathrm{mg}$ FITC in $300 \mu \mathrm{L}$ DMSO was added to a BSA solution (30 mg of BSA in $6 \mathrm{~mL} 0.05 \mathrm{M} \mathrm{NaHCO}_{3} \mathrm{pH}$ 10) in a dropwise fashion. After overnight incubation at room temperature, the excess of FITC was thoroughly removed by two days dialysis against MQ water followed by freeze-drying. 
PLL(15-30 kDa)-graft[2.0]-poly(ethylene glycol (2 kDa)) (PLL-g-PEG) was synthetized following a reported procedure. ${ }^{[37,52]}$ Briefly, a solution containing 0.038 mmol Me-PEG-NHS (with respect to the polymer's molecular weight) in cold HEPES was added to a solution containing $0.143 \mathrm{mmol}$ PLL (with respect to the repeating unit) in chilled HEPES. The reaction was allowed to proceed for $4 \mathrm{~h}$ at $4{ }^{\circ} \mathrm{C}$ under constant stirring. Finally, the reaction mixture was dialyzed for 2 days against MQ water.

Hemoglobin (Hb) Extraction from Bovine Red Blood Cells: Hb was extracted from fresh blood by hypotonic hemolysis following a previous study. ${ }^{[53]}$ Briefly, bovine red blood cells (bRBCs) were first washed with an isotonic saline solution, i.e., $0.9 \% \mathrm{NaCl}(3 \times, 15 \mathrm{~min}, 1500 \mathrm{rcf})$. Next, the pellet containing the bRBCs was mixed with MQ water and toluene at a 1:1:0.4 volume ratio. Following thorough vortexing, the suspension was stored overnight at $4{ }^{\circ} \mathrm{C}$ to allow for the separation of the aqueous and organic phases. Next, the organic phase was removed and the aqueous suspension was spun down (20 min, $8000 \mathrm{rcf}$ ). Following filtration through an ash-free filtration paper, the stroma-free $\mathrm{Hb}$ was collected and stored at $-80{ }^{\circ} \mathrm{C}$ for future use.

Preparation of Hb-Nanoparticles: Nanoparticles fully made of $\mathrm{Hb}$ (Hb-NPs) were prepared using the electrospray instrument BI-LE50AC (I\&L Biosystems Nordic A/S, Denmark) equipped with a co-axial nozzle. The distance between the nozzle tip and the collector was adjusted to $10 \mathrm{~cm}$ and flow rates of 80 and $480 \mu \mathrm{L} \mathrm{h}^{-1}$ were used for the inner $\left(30 \mathrm{mg} \mathrm{mL}^{-1} \mathrm{Hb}\right.$ solution in MQ water) and the outer channels (EtOH solvent), respectively. To obtain a stable cone-jet, the voltage was adjusted to $20 \mathrm{kV}$. The Hb-NPs were collected either with a stationary plate (Hb-NP1) or an EtOH bath (Hb-NP2) connected to a negative voltage generator $(-2 \mathrm{kV})$ at room temperature and ambient humidity. A Table detailing the settings employed for the two formulations can be found in the Supporting Information (Table S1).

Hb-NPs Surface Modification: The as-prepared Hb-NPs (both Hb-NP1 and Hb-NP2) were incubated in a DA solution ( $1 \mathrm{mg} \mathrm{mL}^{-1}$ in TRIS, $30 \mathrm{~min}$, room temperature) followed by 
washing in MQ water (2×, $13000 \mathrm{rcf}, 30 \mathrm{~min})$ to obtain polydopamine (PDA)-coated NPs (Hb/PDA-NPs). Next, to render PEGylated Hb-NPs (Hb/PEG-NPs) the samples were incubated in a PLL- $g$-PEG solution ( $1 \mathrm{mg} \mathrm{mL}^{-1}$ in TRIS, $1 \mathrm{~h}$, room temperature) followed by washing with MQ water (2×, $13000 \mathrm{rcf}, 30 \mathrm{~min})$.

Scanning Electron Microscopy (SEM): The samples were prepared by adding a droplet of NPs suspension (Hb-NPs, Hb/PDA-NPs and Hb/PEG-NPs) on a glass slide which was subsequently left to dry overnight. Next, the samples were sputtered with gold for $20 \mathrm{~s}$ employing a Cressington 208HR High Resolution Sputter Coater (Cressington Scientific Instruments Ltd., UK) and imaged using a TM3030 PLUS Hitachi High-Tech scanning electron microscope (HITACHI, USA) at an operating voltage of $15 \mathrm{kV}$.

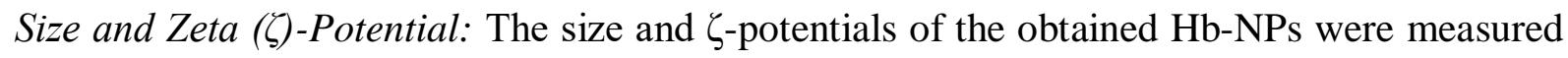
using a nanoparticle tracking analyzer (NTA, ZetaView, Particle Metrix GmbH, Germany). In brief, a suspension of the NPs in MQ water was loaded into the instrument and the NTA measurement was captured at 11 different positions. The size distribution and $\zeta$-potential were obtained by processing the recorded data employing the associated ZetaView 8.05.04 (ZNTA) software.

PEGylation Effect: The PEGylation effect was assessed in terms of protein adsorption onto the NPs. Suspensions of Hb-NPs, Hb/PDA-NPs or Hb/PEG-NPs displaying 100 000 events $\mu \mathrm{L}^{-1}$ (measured by flow cytometry, BD Biosciences, Sparks, MD, USA), were incubated in a $1 \mathrm{mg}$ $\mathrm{mL}^{-1}$ IgG-FITC or $0.5 \mathrm{mg} \mathrm{mL}^{-1}$ BSA-FITC solution in PBS $(200 \mu \mathrm{L})$ at $37^{\circ} \mathrm{C}$ for $4 \mathrm{~h}$ using a thermoshaker (Eppendorff, AG, Germany). After the incubation time, the samples were washed in PBS (2×, $5 \mathrm{~min}, 11000 \mathrm{rcf})$ to remove unbound proteins. The fluorescence intensity due to the bound proteins onto the particles was analyzed by flow cytometry using an excitation 
wavelength of $488 \mathrm{~nm}$ and an emission detection wavelength of $530 \mathrm{~nm}$. At least 20000 events were analyzed in two independent experiments.

Fourier-Transform Infrared (FTIR) Spectroscopy: FTIR analysis was performed using a Perkin Elmer Spectrum 100 FT-IR spectrometer (Perkin Elmer Inc., Wellesley, MA) under ambient conditions. The spectra were collected within the wavelength range of 4000 to $400 \mathrm{~cm}^{-1}$, each one with a resolution of $4 \mathrm{~cm}^{-1}$. Five scans per sample were conducted.

Circular Dichroism (CD) Studies: The CD spectra were recorded on a JASCO J-815 instrument (JASCO, Essex, UK) at room temperature. The following instrument settings were employed: $1 \mathrm{~mm}$ cell length, $1 \mathrm{~nm}$ data interval, $1 \mathrm{~nm}$ band width and $4 \mathrm{~s}$ digital integration time. Free $\mathrm{Hb}$ (40 $\left.\mu \mathrm{g} \mathrm{mL}^{-1}\right), \mathrm{Hb}-\mathrm{NP} 1\left(121 \mu \mathrm{g} \mathrm{mL}^{-1}\right)$ and Hb-NP2 $\left(199 \mu \mathrm{g} \mathrm{mL}^{-1}\right)$ dispersed in MQ water were measured at least three times within the wavelength range of $190-260 \mathrm{~nm}$. All presented spectra were obtained by averaging three different scans, subtracting the corresponding solvent spectrum and, if required, normalizing to an ellipticity value of -1 at $222 \mathrm{~nm}$.

Oxygen Binding and Releasing Studies: The UV-Vis spectra of PEGylated (Hb/PEG-NPs) and non-PEGylated (Hb-NPs) samples were measured on a UV-2600 UV-vis Spectrophotometer (Shimadzu, Japan) in the wavelength range of $350-650 \mathrm{~nm}$. The different samples were dispersed in MQ water containing a pinch of SDT. To obtain samples with deoxygenated Hb (deoxy-Hb), nitrogen gas $\left(\mathrm{N}_{2}\right)$ was allowed to flow over the samples for $10 \mathrm{~min}$ followed by recording the UV-vis spectrum. Next, the ability of the particles to bind oxygen was evaluated by purging with oxygen gas $\left(\mathrm{O}_{2}\right)$ for $10 \mathrm{~min}$ followed by recording the $\mathrm{UV}$-vis spectrum of the oxygenated $\mathrm{Hb}$ (oxy- $\mathrm{Hb}$ ). Subsequent alternating purging of the samples with $\mathrm{N}_{2}$ and $\mathrm{O}_{2}$ was conducted to assess the ability of the samples to reversibly bind and release oxygen.

Antioxidant Protection of $\mathrm{Hb}$ by the PDA Coating: The UV-vis spectra of Hb-NPs before and after adding $5 \mathrm{mM} \mathrm{H} \mathrm{H}_{2} \mathrm{O}_{2}$ were recorded with the UV-vis $2600 \mathrm{UV}$-vis spectrophotometer. The 
decrease of the characteristic Soret peak of oxy-Hb was used to monitor the conversion of oxy$\mathrm{Hb}$ into metHb.

Hemolysis Rate Test: Hemolysis rate test was conducted to investigate the blood compatibility of the obtained bare (Hb-NPs), PDA-coated (Hb/PDA-NPs) and PEGylated (Hb/PEG-NPs) NPs. First, whole blood from healthy volunteers was collected in heparin (anticoagulant) containing tubes. The blood was washed in PBS (3×, $1000 \mathrm{rcf}, 15 \mathrm{~min})$ and the pellet was resuspended in $50 \mathrm{~mL}$ of PBS to obtain diluted blood. The different NPs were dispersed in PBS $\left(1 \mathrm{mg} \mathrm{mL}{ }^{-1}\right)$ and incubated at $37^{\circ} \mathrm{C}$ for $30 \mathrm{~min}$. Next, the NPs suspensions were incubated with the diluted blood at $1: 1 \mathrm{v} / \mathrm{v}$ ratio at $37{ }^{\circ} \mathrm{C}$ for $60 \mathrm{~min}$. A thermoshaker (Eppendorff, AG, Germany) with a shaking speed of $250 \mathrm{rpm}$ was employed. Diluted blood in MQ water or PBS were used as positive and negative controls, respectively. After the incubation time, the samples were spun down (1000 rcf, $10 \mathrm{~min})$ and the pellets were collected for morphology observation while the supernatants were further centrifuged (13 $000 \mathrm{rcf}, 10 \mathrm{~min})$. The new supernatants were collected and the absorbance was recorded at $540 \mathrm{~nm}$. The hemolysis rate was calculated as follows: hemolysis rate $(\%)=($ experimental value - negative control value $) /($ positive control value - negative control value) $\times 100$.

The pellets were resuspended in PBS and the morphology of the blood cells was assessed by differential interference contrast (DIC) microscopy. An Olympus Inverted IX83 microscope equipped with a $63 \times$ oil immersion objective was employed.

Cell Viability: The mouse macrophage Raw 264.7 cell line was cultured in DMEM supplemented with $10 \%(\mathrm{v} / \mathrm{v}) \mathrm{FBS}, 1 \%(\mathrm{v} / \mathrm{v})$ penicillin/streptomycin $\left(10000 \mathrm{U} \mathrm{mL}^{-1}\right.$ and $10 \mu \mathrm{g}$ $\mathrm{mL}^{-1}$, respectively) at $37{ }^{\circ} \mathrm{C}$ in a humidified incubator and $5 \% \mathrm{CO}_{2}$. The endothelial HUVEC cell line was cultured in ECM supplemented with 5\% (v/v) FBS and 1\% (v/v) endothelial cell growth supplements and 1\% (v/v) penicillin/streptomycin $\left(10000 \mathrm{U} \mathrm{mL}^{-1}\right.$ and $10 \mu \mathrm{g} \mathrm{mL}^{-1}$, respectively) at $37{ }^{\circ} \mathrm{C}$ and $5 \%(\mathrm{v} / \mathrm{v}) \mathrm{CO}_{2}$. The cells were cultured in $\mathrm{T} 75$ flasks and the cell 
media was exchanged 2 - 3 times per week. Only cells between passages 5 and 12 (for RAW 264.7) and between passages 3 and 10 (for HUVEC) were used in all experiments. While a cell scraper was employed to detach sub-confluent RAW 264.7 cells from the culture flask, subconfluent HUVEC cells were detached from the culture flask by adding trypsin ( $3 \mathrm{~mL})$. Both RAW 264.7 and HUVEC cells were aspirated and re-suspended in cell media. Appropriate aliquots of the cell suspension were added into new T75 culture flasks. All cell experiments were conducted in triplicate and at least two independent experiments were carried out.

For cell viability assays, 96-well plates were used and the RAW 264.7 and HUVEC cells were seeded in $200 \mu \mathrm{L}$ of full media at a density of 30000 and 15000 cells per well, respectively, and allowed to attach for $24 \mathrm{~h}$. Next, the cells were washed in PBS $(3 \times, 200 \mu \mathrm{L})$ and $200 \mu \mathrm{L}$ of the as-prepared particle solutions were added to the different wells at a concentration of $0.5 \mathrm{mg}$ $\mathrm{mL}^{-1}$. Following incubation for 4 and $24 \mathrm{~h}$, respectively, the media of the wells was aspired carefully and the cells were washed in PBS $(3 \times, 200 \mu \mathrm{L})$. Next, $100 \mu \mathrm{L}$ of fresh full cell media containing PrestoBlue $(10 \% \mathrm{v} / \mathrm{v})$ was added to each well and the cells were further incubated for $1 \mathrm{~h}$ at $37{ }^{\circ} \mathrm{C}$ and $5 \% \mathrm{CO}_{2}$. Next, the supernatants were transferred to a new 96-well plate and analyzed using a TECAN Spark multimode plate reader (Tecan Group Ltd., Maennendorf, Switzerland) by measuring the fluorescence intensity of the reduced resaruzin product at 615 $\mathrm{nm}$ using the excitation wavelength at $535 \mathrm{~nm}$. Cells only and cell media only were used a positive and negative controls, respectively. The normalized cell viability was calculated as follows: normalized cell viability $=($ sample value - negative control value $) /($ positive control value - negative control value) $\times 100$.

Statistical analysis: A Dunnett's multiple comparison test $\left({ }^{*} \mathrm{p} \leq 0.05 ; * * \mathrm{p} \leq 0.001,{ }^{* * *} \mathrm{p} \leq\right.$ 0.0001) was employed using GraphPad Prism (8.1.0 (325)) software to assess the statistical differences between the different conditions. A one-way analysis of variance with a confidence level of $95 \%(\alpha=0.005)$ was employed. 


\section{Supporting Information}

Supporting Information is available from the Wiley Online Library or from the author.

\section{Acknowledgements}

This work was supported by the Danish Council for Independent Research (Grant No. 611100298B).

\section{References}

[1] W.-L. Liu, T. Liu, M.-Z. Zou, W.-Y. Yu, C.-X. Li, Z.-Y. He, M.-K. Zhang, M.-D. Liu, Z.-H. Li, J. Feng, et al., Adv. Mater. 2018, 30, 1802006.

[2] Y. Jia, Y. Cui, J. Fei, M. Du, L. Dai, J. Li, Y. Yang, Adv. Funct. Mater. 2012, 22, 1446.

[3] W. H. O. E. Group, Vox Sang. 2012, 103, 337.

[4] N. Hamasaki, M. Yamamoto, Vox Sang. 2000, 79, 191.

[5] N. Dhingra, Vox Sang. 2002, 83, 173.

[6] Y. Jia, Y. Cui, J. Fei, M. Du, L. Dai, J. Li, Y. Yang, Adv. Funct. Mater. 2012, 22, 1446.

[7] A. I. Alayash, Nat. Rev. Drug Discovery 2004, 3, 152.

[8] C. Natanson, S. J. Kern, P. Lurie, S. M. Banks, S. M. Wolfe, J. Am. Med. Assoc. 2008, 299, 2304.

[9] J. Simoni, G. Simoni, D. E. Wesson, M. Feola, Curr. Drug Discovery Technol. 2012, 9, 173.

[10] Z. Pan, T. Wu, Y. Liu, C. Yu, N. Bao, H. Gu, Surfaces and Interfaces 2017, 6, 50.

[11] S. A. Gould, E. E. Moore, D. B. Hoyt, J. M. Burch, J. B. Haenel, J. Garcia, R. DeWoskin, G. S. Moss, J. Am. Coll. Surg. 1998, 187, 113.

[12] S. Wang, F. Yuan, G. Chen, K. Tu, H. Wang, L.-Q. Wang, RSC Adv. 2014, 4, 52940.

[13] J. S. Jahr, A. Sadighi, L. Doherty, A. Li, H. Won Kim, Chem. Biochem. Oxyg. Ther. from Transfus. to Artif. blood 2011, 301.

[14] A. I. Alayash, Trends Biotechnol. 2014, 32, 177.

[15] P. Cabrales, G. Sun, Y. Zhou, D. R. Harris, A. G. Tsai, M. Intaglietta, A. F. Palmer, J. 
Appl. Physiol. 2009, 107, 1548.

[16] R. M. J. Palmer, A. G. Ferrige, S. Moncada, Nature 1987, 327, 524.

[17] M. M. T. Jansman, L. Hosta-Rigau, Adv. Colloid Interface Sci. 2018.

[18] S. Kaneda, T. Ishizuka, H. Goto, T. Kimura, K. Inaba, H. Kasukawa, Artif. Organs 2009, 33, 146.

[19] S. Rameez, H. Alosta, A. F. Palmer, Bioconjugate Chem. 2008, 19, 1025.

[20] N. Cedrati, F. Bonneaux, P. Labrude, P. Maincent, Artif. Cells, Blood Substitutes, Immobilization Biotechnol. 1997, 25, 457.

[21] R. Sridhar, S. Ramakrishna, Biomatter 2013, 3, DOI 10.4161/biom.24281.

[22] A. C. Mendes, K. Stephansen, I. S. Chronakis, Food Hydrocoll. 2017, 68, 53.

[23] A. C. Mendes, I. S. Chronakis, Adv. Food Nutr. Res. 2019, 88, 167.

[24] A. Arinstein, M. Burman, O. Gendelman, E. Zussman, Nat. Nanotechnol. 2007, 2, 59.

[25] M. Komiyama, K. Yoshimoto, M. Sisido, K. Ariga, Bull. Chem. Soc. Jpn. 2017, 90, 967.

[26] S. Mitragotri, J. Lahann, Nat. Mater. 2009, 8, 15.

[27] J. A. Champion, A. Walker, S. Mitragotri, Pharm. Res. 2008, 25, 1815.

[28] S. Rameez, A. F. Palmer, Langmuir 2011, 27, 8829.

[29] J. H. Ryu, P. B. Messersmith, H. Lee, ACS Appl. Mater. Interfaces 2018, 10, 7523.

[30] O. Baidukova, Q. Wang, S. Chaiwaree, D. Freyer, A. Prapan, R. Georgieva, L. Zhao, H. Bäumler, Artif. Cells, Nanomed., Biotechnol. 2018, 46, S693.

[31] X. Liu, L. H. Nielsen, H. Qu, L. P. Christensen, J. Rantanen, M. Yang, Eur. J. Pharm. Biopharm. 2019, 136, 240.

[32] A. Arinstein, M. Burman, O. Gendelman, E. Zussman, Nat. Nanotechnol. 2007, 2, 59.

[33] I. Lalezari, P. Lalezari, C. Poyart, M. Marden, J. Kister, B. Bohn, G. Fermi, M. F. Perutz, Biochemistry 1990, 29, 1515.

[34] H. Sakai, Y. Masada, S. Takeoka, E. Tsuchida, J. Biochem. 2002, 131, 611. 
[35] Y. Xiong, Z. Z. Liu, R. Georgieva, K. Smuda, A. Steffen, M. Sendeski, A. Voigt, A. Patzak, H. Bäumler, ACS Nano 2013, 7, 7454.

[36] S. J. Watt, M. M. Sheil, J. L. Beck, P. Prosselkov, G. Otting, N. E. Dixon, J. Am. Soc. Mass Spectrom. 2007, 18, 1605.

[37] M. J. York-Duran, P. K. Ek, M. Godoy-Gallardo, L. Hosta-Rigau, Colloids Surf., B 2018, 171, 427.

[38] M. Hautreux, N. Hue, A. D. F. de Kerdaniel, A. Zahir, V. Malec, O. Laprévote, Int. J. Mass Spectrom. 2004, 231, 131.

[39] J. S. Suk, Q. Xu, N. Kim, J. Hanes, L. M. Ensign, Adv. Drug Delivery Rev. 2016, 99, 28.

[40] K. Parmar, J. K. Patel, in Surf. Modif. Nanoparticles Target. Drug Deliv., Springer International Publishing, Cham, 2019, pp. 221-236.

[41] K. Ogawara, K. Furumoto, S. Nagayama, K. Minato, K. Higaki, T. Kai, T. Kimura, J. Controlled Release 2004, 100, 451.

[42] N. Bertrand, J.-C. Leroux, J. Controlled Release 2012, 161, 152.

[43] J. T. Pelton, L. R. McLean, Anal. Biochem. 2000, 277, 167.

[44] N. J. Greenfield, Nat. Protoc. 2006, 1, 2876.

[45] R. Liu, P. Qin, L. Wang, X. Zhao, Y. Liu, X. Hao, J. Biochem. Mol. Toxicol. 2010, 24, 66.

[46] A. J. Miles, B. A. Wallace, Chem. Soc. Rev. 2016, 45, 4859.

[47] M. Lu, C. Zhao, Q. Wang, G. You, Y. Wang, H. Deng, G. Chen, S. Xia, J. Zhao, B. Wang, et al., Colloids Surf., B 2016, 139, 171.

[48] W. L. Stoppel, S. C. Roberts, in Eng. Biomater. Regen. Med., Springer New York, New York, NY, 2012, pp. 41-86.

[49] R. Luo, L. Tang, S. Zhong, Z. Yang, J. Wang, Y. Weng, Q. Tu, C. Jiang, N. Huang, ACS Appl. Mater. Interfaces 2013, 5, 1704. 
[50] W.-B. Tsai, W.-T. Chen, H.-W. Chien, W.-H. Kuo, M.-J. Wang, Acta Biomater. 2011, $7,4187$.

[51] L. Hosta-Rigau, B. Städler, Mol. Pharm. 2013, 10, 2707.

[52] M. Godoy-Gallardo, C. Labay, V. D. V.D. Trikalitis, P. J. P. J. Kempen, J. B. J. B. Larsen, T. L. Andresen, L. Hosta-Rigau, ACS Appl. Mater. Interfaces 2017, 9, 15907.

[53] W. G. Zijlstra, A. Buursma, Comp. Biochem. Physiol. - B Biochem. Mol. Biol. 1997, $118,743$.

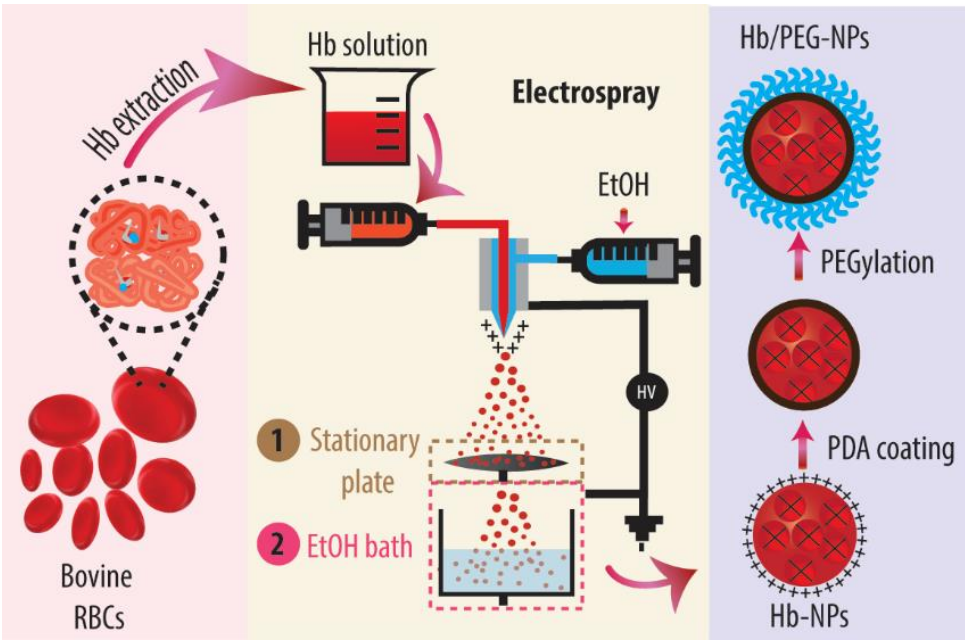

Scheme 1. Illustration of the assembly process. Hemoglobin $(\mathrm{Hb})$ is first extracted from bovine red blood cells (RBCs). Hb-based nanoparticles (Hb-NPs) are prepared by the electrospray technique. In particular, a solution of $\mathrm{Hb}$ in water is electrosprayed and the resulting $\mathrm{Hb}-\mathrm{NPs}$ are collected either in a stationary plate (Hb-NP1) or an ethanol (EtOH) bath (Hb-NP2). An antioxidant poly(dopamine) (PDA) coating is deposited which also allows for further surface modification with poly(ethylene glycol) (PEG). 
a) $\mathrm{Hb}-\mathrm{NPS}$
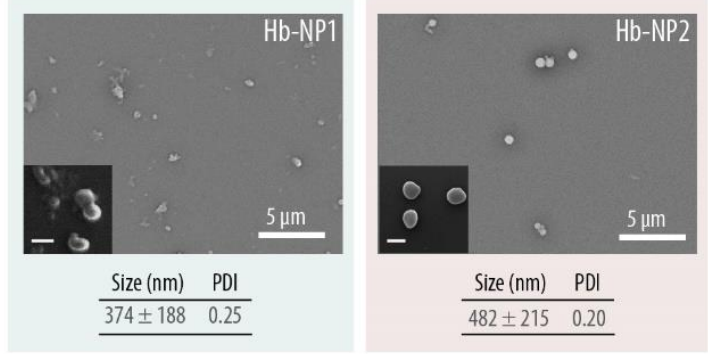

b) $\mathrm{Hb} / \mathrm{PDA}-\mathrm{NPs}$

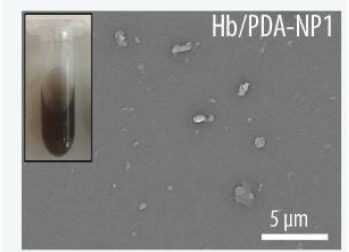

$$
\begin{array}{cc}
\text { Size }(\mathrm{nm}) & \text { PDI } \\
\hline 438 \pm 187 & 0.18 \\
\hline
\end{array}
$$

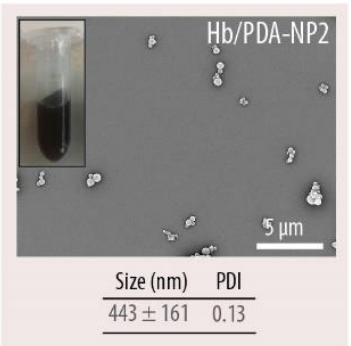

c) PEGylated Hb-NPs

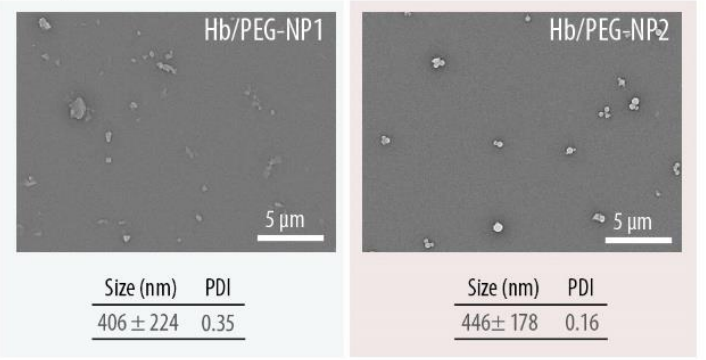

d)

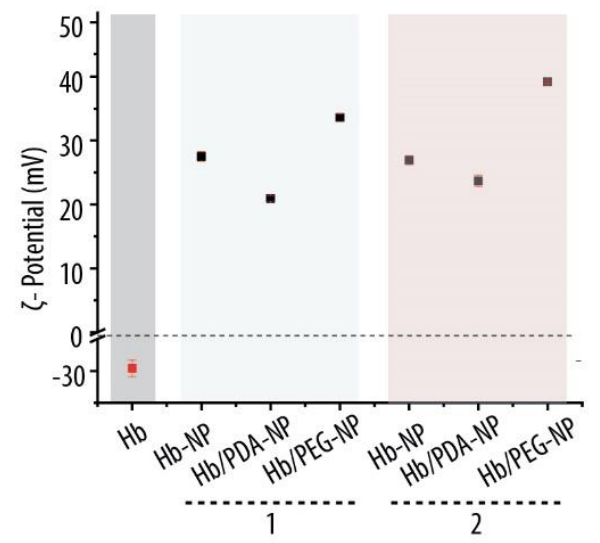

Figure 1. Scanning electron microscopy images of bare Hb-NPs (a), polydopamine (PDA)coated Hb/PDA-NPs (b) and PEGylated Hb/PEG-NPs (c). Size and polydispersity index (PDI)

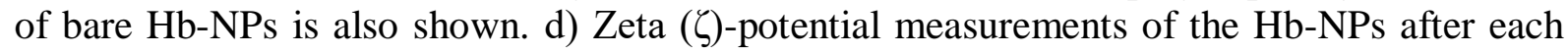
deposition step. The Hb-NPs have been prepared employing two different collection methods: NP1: a stationary plate collector or NP2: an ethanol bath. The scale bar for the inset image in figure (a) is $500 \mathrm{~nm}$. 


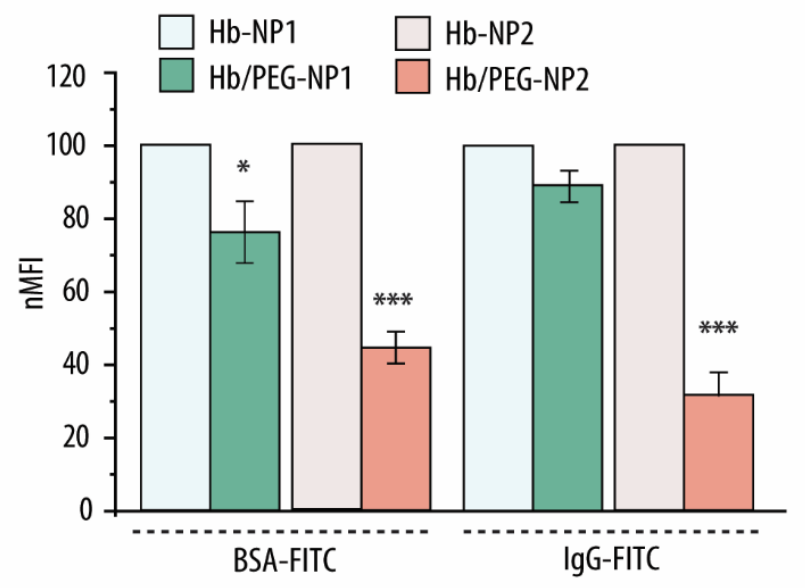

Figure 2. Normalized mean fluorescence intensity (nMFI) of bare Hb-NPs, polydopamine (PDA)-coated Hb/PDA-NPs and PEGylated Hb/PEG-NPs upon incubation with fluorescently labelled bovine serum albumin (BSA-FITC) or immunoglobulin G (IgG-FITC). Two different types of Hb-NPs have been fabricated using different collection methods: NP1: a stationary plate (green columns) or NP2: an ethanol bath (pink columns). 

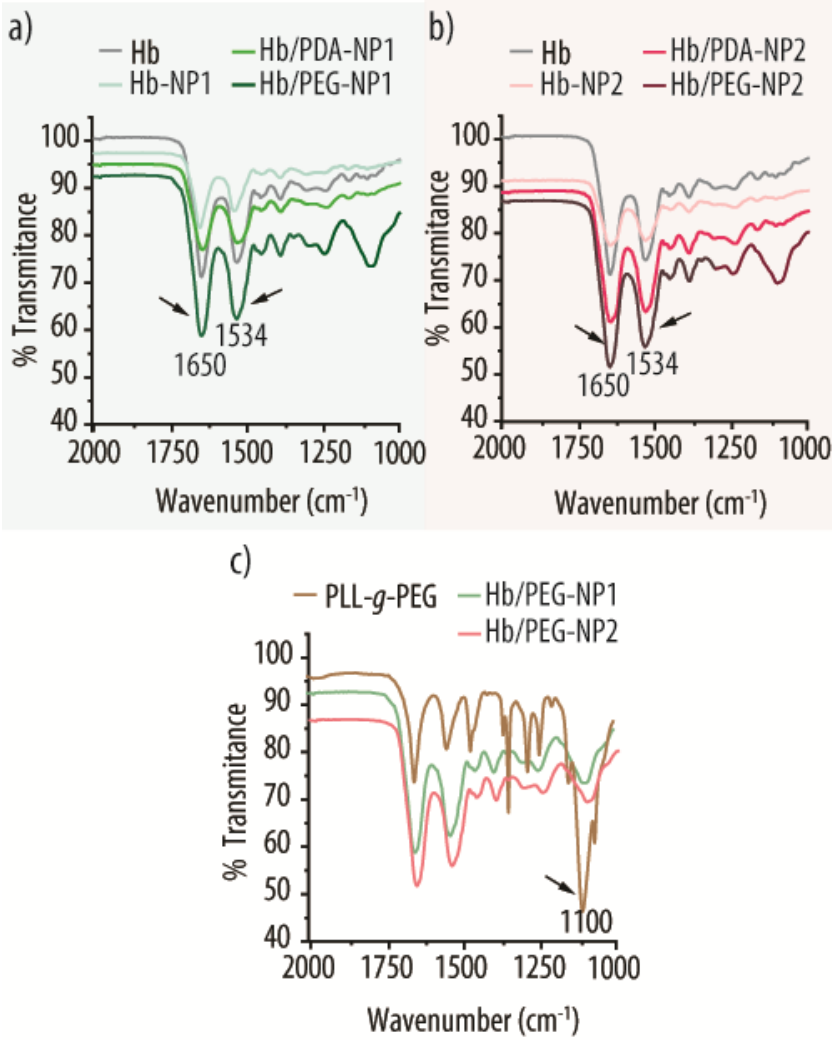

Figure 3. Fourier-transform infrared (FTIR) spectra of the bare Hb-NPs, polydopamine (PDA)coated $\mathrm{Hb} / \mathrm{PDA}-\mathrm{NPs}$ and PEGylated Hb/PEG-NPs as compared to native $\mathrm{Hb}$. Two different types of Hb-NPs have been fabricated using different collection methods: NP1: a stationary plate (a) or NP2: an ethanol bath (b). c) FTIR spectra of Hb/PEG-NPs fabricated using the two different collection methods as compared to poly( $L$-lysine)-graft-poly(ethylene glycol) (PLL$g$-PEG). The arrow pointing at $1650 \mathrm{~cm}^{-1}$ denotes the Amide I peak; the arrow pointing at 1534 $\mathrm{cm}^{-1}$ denotes the Amide II group and the arrow pointing to $1100 \mathrm{~cm}^{-1}$ denotes the ether peak. 

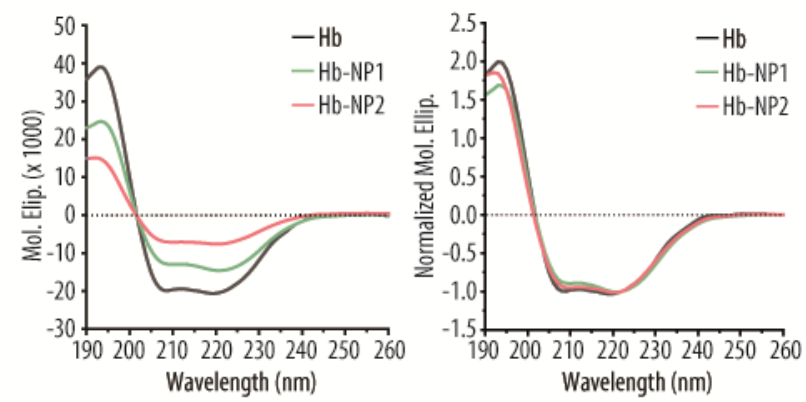

Figure 4. Circular dichroism spectra of a freshly extracted $\mathrm{Hb}$ solution and $\mathrm{Hb}-\mathrm{NPs}$ fabricated using either a plate collector method (Hb-NP1) or an ethanol bath method (Hb-NP2), respectively. The $\mathrm{CD}$ spectra on the right side have been normalized to an ellipticity value of 1 at $222 \mathrm{~nm}$. 


$$
\begin{array}{ll}
- \text { Initial } & -\mathrm{O}_{2} \text { - Cycle } 1-\mathrm{O}_{2} \text { - Cycle 2 } \\
-\mathrm{N}_{2} \text { - Cycle 1 }-\mathrm{N}_{2} \text { - Cycle 2 } &
\end{array}
$$

a)

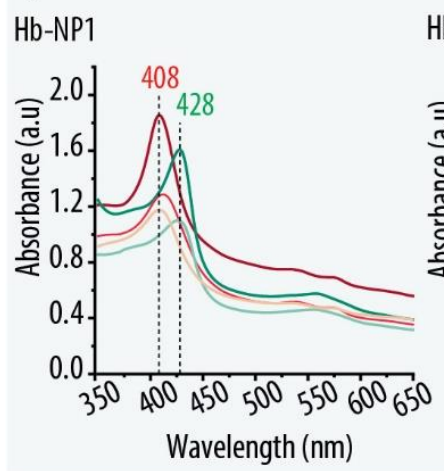

Hb/PEG-NP1

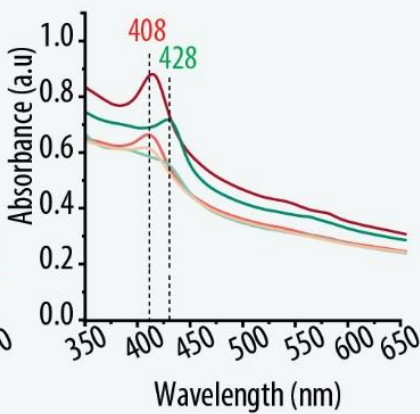

b)

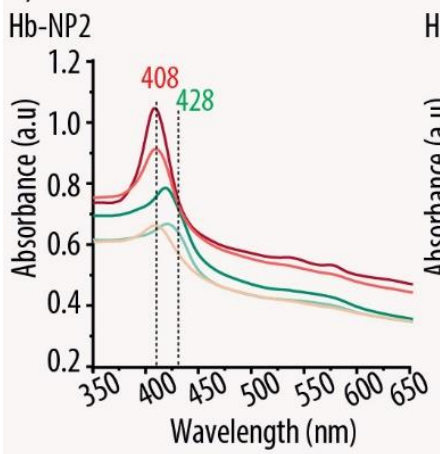

Hb/PEG-NP2

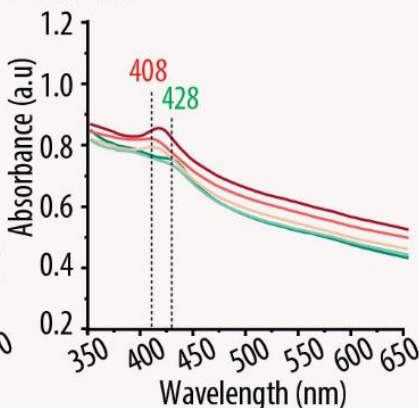

Figure 5. UV-vis absorption spectra of bare $\mathrm{Hb}-\mathrm{NPs}$ and PEGylated Hb/PEG-NPs after successively feeding with oxygen $\left(\mathrm{O}_{2}\right)$ or nitrogen $\left(\mathrm{N}_{2}\right)$ gas.

Two different types of Hb-NPs have been fabricated using different collection methods: NP1: a stationary plate (a) or NP2: an ethanol bath (b). 

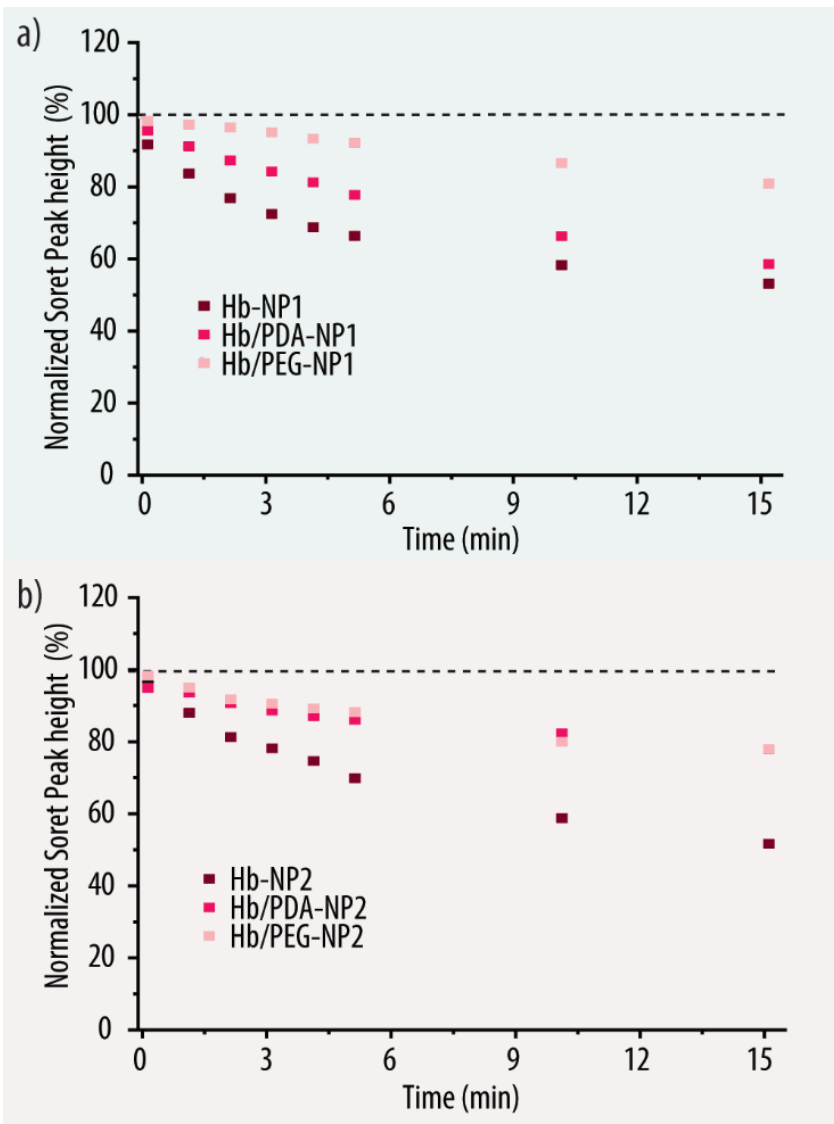

Figure 6. Soret peak height over time for bare Hb-NPs, polydopamine (PDA)-coated Hb/PDANPs and PEGylated Hb/PEG-NPs. Two different types of Hb-NPs have been fabricated using different collection methods: 1: a stationary plate (a) or 2: an ethanol bath (b). 
a) i)

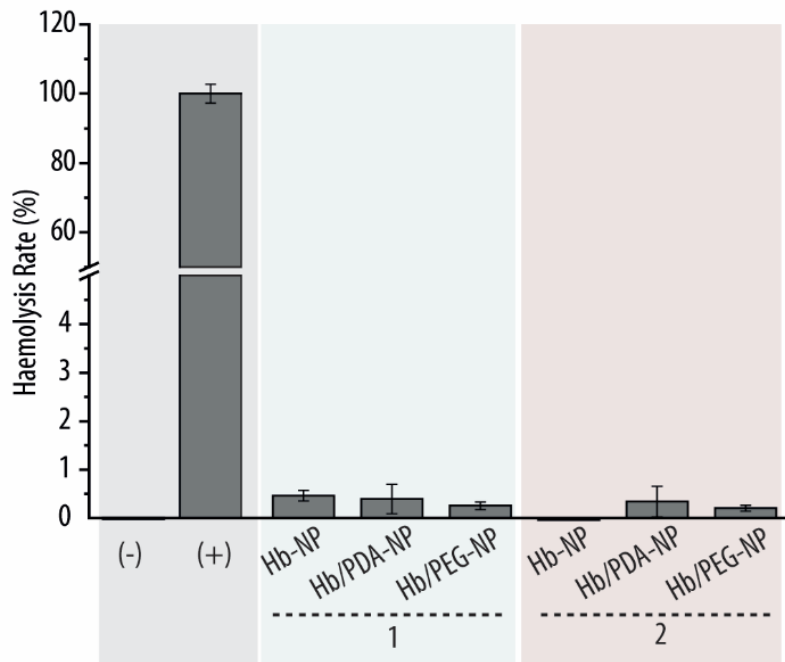

b) i) HUVEC, $4 \mathrm{~h}$

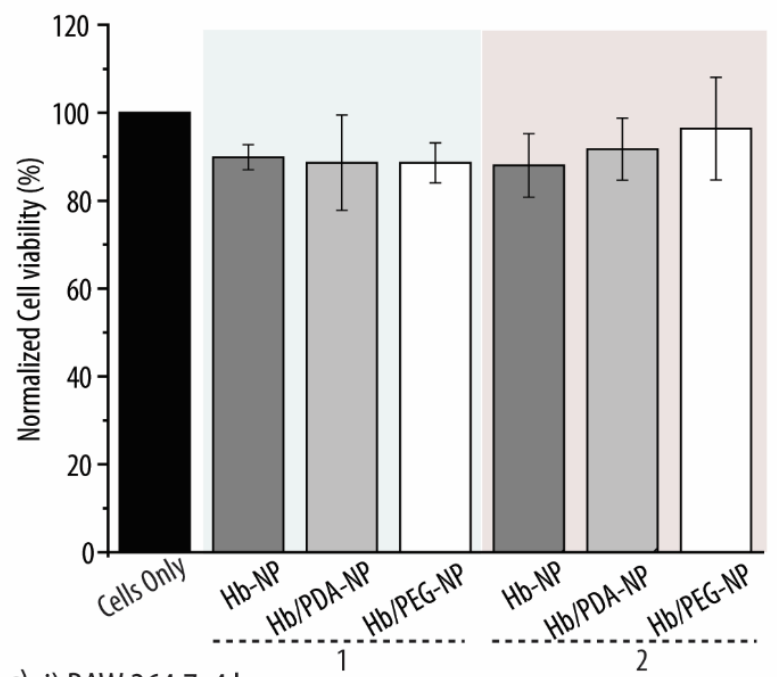

c) i) RAW $264.7,4 \mathrm{~h}$

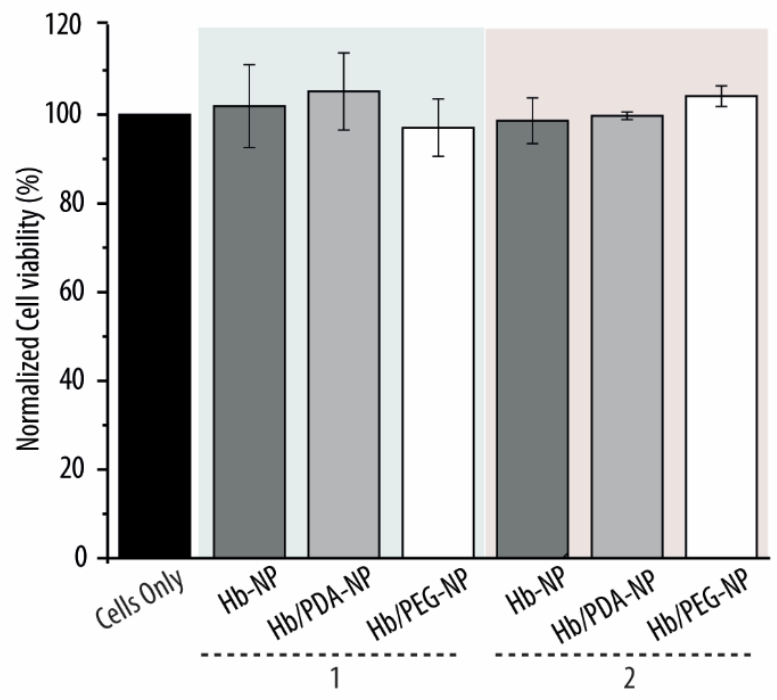

ii)

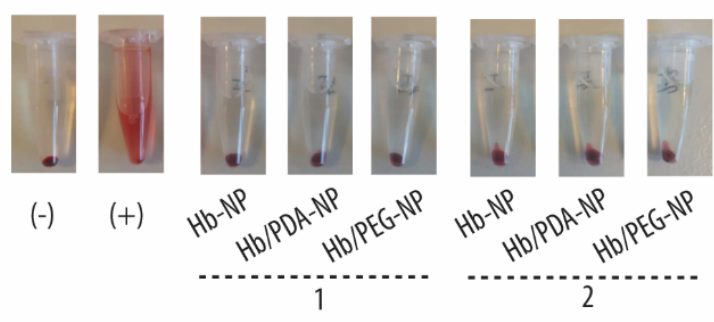

ii) HUVEC, $24 \mathrm{~h}$

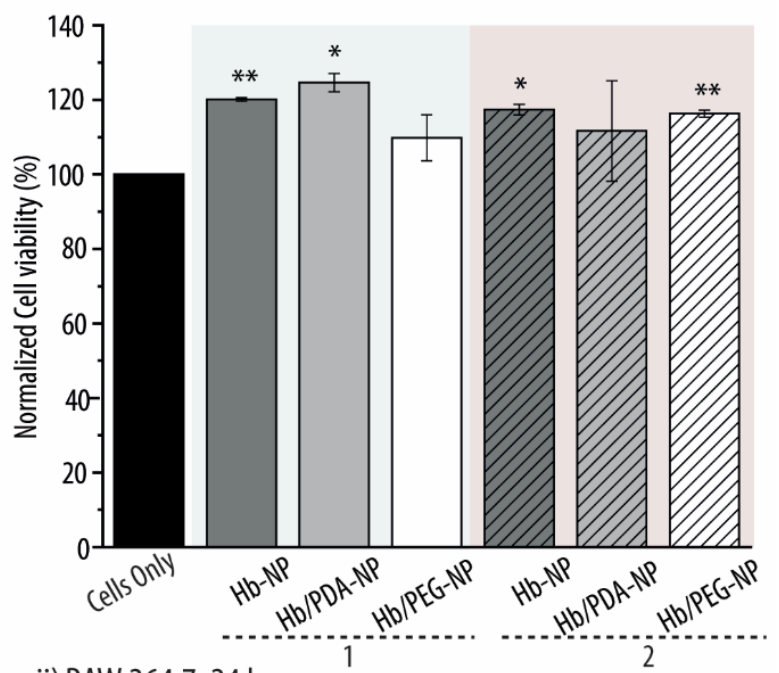

ii) RAW 264.7, $24 \mathrm{~h}$

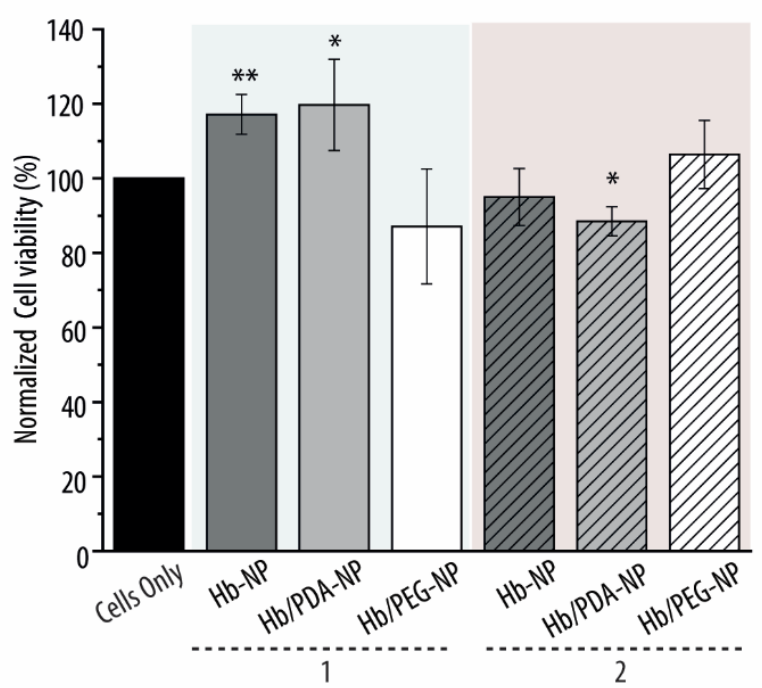

Figure 7. a) Hemolysis rate (i) and photographic images (ii) of the negative control (-), positive control (+) and the different samples: uncoated Hb-NPs, polydopamine (PDA)-coated Hb/PDANP and PEGylated Hb/PEG-NPs obtained by two different collection methods (1: stationary plate or 2: ethanol bath). Normalized cell viability readings of HUVEC (endothelial cells) (b) and RAW 264.7 (macrophages) (c) cells following exposure to bare Hb-NPs, Hb/PDA-NP and 
Hb/PEG-NPs obtained by the two different collection methods. The cells were incubated for either $4 \mathrm{~h}$ (i) or $24 \mathrm{~h}$ (ii). 